\title{
La lateralidad en el Karate: estudio centrado en la praxis de los entrenadores y entrenadoras \\ Laterality in Karate: study focused on the praxis of coaches
}

Nerea Estrada-Marcén, Alejandro López-Rubio, Jaime Casterad-Seral

Universidad de Zaragoza (España)

\begin{abstract}
Resumen. La lateralidad es un fenómeno ampliamente estudiado, pero aún no se han determinado claramente qué factores intervienen en su desarrollo. No obstante, fundamentalmente durante la niñez, varios estudios han demostrado que la dominancia lateral se puede llegar a entrenar y modificar, y en diversas disciplinas deportivas la dominancia lateral resulta ser un factor clave en el rendimiento, y se contempla como tal en los entrenamientos que se plantean. Nuestro objetivo es examinar la percepción de los entrenadores del tratamiento de la lateralidad en el entrenamiento del karate. Hemos empleado metodología cuantitativa de corte descriptivo, llevándose a cabo un estudio transversal basado en la muestra, aplicando un cuestionario diseñado ad hoc. Han accedido a participar en el estudio 72 entrenadores/as en activo. El ambidextrismo es el tipo de lateralidad que más ventajas aporta a nivel competitivo según los entrenadores/as. El segmento en el que es más común que los deportistas sufran un cambio de lateralidad forzada es el pie. Dicha modificación suele producirse mayoritariamente en aquellas personas que son diestras, con el objetivo de que cambien a una dominancia lateral zurda. La mayoría de entrenadores / as que intentar cambiar la lateralidad de sus deportistas lo hace a partir de los 8 años, pareciendo éste un momento óptimo para realizar el cambio, y normalmente cuando éstos se encuentran en nivel competitivo nacional. Los entrenadores/as «casi siempre» realizan un trabajo bilateral equilibrado en kumite y en kata, considerando impor tante mantener este balance.
\end{abstract}

Palabras clave: Lateralidad, dominancia lateral, karate, entrenadores, karateka.

\begin{abstract}
Laterality is a widely studied phenomenon, but the factors involved in its development have not yet been clearly determined. However, mainly during childhood, several studies have shown that lateral dominance can be trained and modified. In various sports disciplines lateral dominance turns out to be a key factor in performance, so it is considered as such in training. Our objective is to examine the coaches' perception of the laterality treatment in karate training. We have used a descriptive quantitative methodology, carrying out a cross-sectional study based on the sample, applying an ad hoc designed questionnaire. 72 active coaches have agreed to participate in the study. Ambidextrism is the type of laterality that provides the most advantages at a competitive level according to coaches. The segment in which it is most common for athletes to undergo a forced lateral change is the foot. Such modification usually occurs mainly in those people who are right-handed, with the aim that they change to a left-handed lateral dominance. Most coaches who try to change the laterality of their athletes, do so when they are more than 7 years old, and usually when they are at a national competitive level. Coaches «almost always» perform balanced bilateral work in kumite and kata, considering it important to maintain this balance.
\end{abstract}

Keywords: Laterality, lateral dominance, karate, coaches, karateka.

\section{Introducción}

El concepto de lateralidad posee diferentes puntos de vista a la hora de definirlo y de entender de qué se trata. Como consecuencia, no hay un consenso universal en lo que se refiere a cómo definir la lateralidad. Por una parte, Pieron (1968, citado en Lerbert, 1977) propone una definición de carácter cuantitativo, en la cual describe la lateralidad como un predominio de un lado del cuerpo en manos y ojos, estableciendo, de este modo, una diferencia entre diestros/zurdos y manua-

Fecha recepción: 19-10-21. Fecha de aceptación: 11-01-22

Nerea Estrada-Marcén

nereaes@unizar.es les/oculares. Por otra parte, Hildreth (1949, citado en Lerbert, 1977) defiende una postura cualitativa, puesto que afirma que la lateralidad depende del grado de habilidad con que utilizamos cada lado corporal. Asimismo, Harris (1961, citado en Lerbert, 1977) elabora otra definición en la cual combina el carácter cualitativo de Hildreth, puesto que entiende la lateralidad como la preferencia de utilización y una mayor maestría en cuanto a aptitudes de un lado corporal frente a otro, y el cuantitativo de Pieron, ya que explica que la lateralidad está presente en manos, pies y ojos. Otros investigadores dan importancia al efecto de los hemisferios cerebrales en su definición del concepto de lateralidad. En esta línea, Sainburg (2016) aboga por un modelo que propone que el hemisferio izquierdo está especializado 
en realizar procesos predictivos que requieren trayectorias de movimiento precisas y eficientes en circunstancias ambientales mecánicamente estables, mientras que el hemisferio derecho se especializa en generar mecanismos de control dinámico que permita realizar movimientos sólidos en situaciones mecánicamente inestables e impredecibles (lo cual se denomina dominancia dinámica). El término dominante, según Ferré e Irabau (2002), pasa a ser referente, puesto que, aunque existe un hemisferio que se encarga de dirigir las acciones, ambos intervienen en mayor o menor medida. Existe un hemisferio dominante/preferente pero no hay ningún hemisferio que esté siendo dominado por el otro. De hecho, el cerebro posee un elemento ubicado entre ambos hemisferios que posibilita dicha interconexión, que se denomina cuerpo calloso y que se encarga principalmente de transmitir información (Bolte, 2009).

Aunque en el proceso de lateralización influye el factor genético, lejos de no poder modificar la lateralidad tras nacer, existen numerosos estudios que afirman que sí que es posible mediante el ejercicio y el aprendizaje motor (Bilbao y Oña, 2000; Haaland y Hoff, 2003; Kumar y Mandal, 2005; Teixeira, Silva y Carvalho, 2003; Van Mier, Perlmutter y Petersen, 2004; Judge y Stirling, 2003). Como el deporte es un fenómeno en el que continuamente se producen aprendizajes motores, el ejercicio se convierte en un canal mediante el cual se pueden implementar técnicas y estrategias que permitan modificar las conductas motrices de los deportistas y, en definitiva, la lateralidad. Señalan Bilbao y Oña (2000) que la administración de reforzamiento positivo, la aportación de feedback extrínseco o la utilización de procedimientos de extinción de conductas, son estrategias efectivas para inducir una modificación en la lateralidad de los deportistas. Además de saber que existen estrategias para modificar la lateralidad, también es interesante conocer el concepto de la transferencia bilateral, que es como denomina Magill (1993, citado en Bejarano y Naranjo, 2014) a un hallazgo que obtiene en su investigación. Dicha transferencia bilateral, consiste en que al entrenar tanto el lado dominante como el no-dominante, se puede mejorar el rendimiento con ambas extremidades e, incluso si se trabaja de manera aislada el lado no-dominante, se logra una mejora en ambos, la cual puede ser incluso mayor que si se trabaja el lado dominante también. Este concepto podría ser relevante a la hora de diseñar y llevar a cabo sesiones de entrenamiento deportivo, puesto que el hecho de poder entrenar con un lado del cuerpo de manera que el opuesto reciba ganancias, puede suponer un gran avance en el rendimiento deportivo y en la capacidad de planificación de los entrenadores/as, ya que se podrían seguir generando adaptaciones incluso en miembros con lesiones. Refiriéndonos de forma concreta al rendimiento deportivo, cada vez se hace más evidente que la lateralidad del sujeto es un factor fundamental para un rendimiento óptimo, pudiéndose ver cómo en determinados deportes una determinada lateralidad puede suponer una ventaja decisiva para la competición (Bejarano y Naranjo, 2014).

Si nos referimos específicamente a la importancia del dominio lateral en Artes marciales, tenemos estudios realizados en Judokas que afirman que la lateralidad de las extremidades superiores e inferiores muestra una correlación significativa con la elección de las direcciones de ataque dominantes en el combate (Sterkowicz, Lech y Blecharz, 2010). Este mismo trabajo concluye que los atletas zurdos muestran significativamente mejores posibilidades de ganar medallas en comparación con los diestros, y alude a otros estudios que evidencian que en Judo la transferencia de técnicas de movimiento de un lado del cuerpo a otro aumenta la efectividad del atleta y le hace ganar, tomando al oponente por sorpresa (Starosta, 2003, citado en Sterkowicz et al., 2010). Y en otro trabajo en Judokas (Sogabe, SterkowiczPrzybycieñ, Maehara, Sasaki, Sterkowicz, 2015), los que emplean el lado izquierdo en su postura dominante en el combate se caracterizan por un perfil más simétrico (más porcentaje igualado de técnicas utilizadas en diferentes direcciones de ataque en comparación con los que emplean el lado derecho), siendo menos predecibles en sus acciones, lo que favorecería su éxito durante un combate de judo, como avalan otros estudios (Franchini, Sterkowicz, Meira, Ferreira-Gomes y Tani, 2008; Mikheev, Mohr, Afanasiev, Landis y Thut, 2002). Cuando nos referimos a las Artes marciales en general, la literatura ha mostrado una relación entre lateralidad y una sobrerrepresentación de deportistas zurdos en determinados deportes, y especialmente en deportes uno contra uno (Dopico-Calvo, Iglesias-Soler, Morenilla, Giráldez, Santos y Ardá, 2016), cuya principal explicación es una mayor probabilidad de éxito.

En el caso concreto del Karate, esta disciplina está compuesta por diferentes modalidades (kata individual, kata por equipos y kumite o combate interindividual), las cuales requieren un dominio lateral equilibrado de todos los segmentos corporales para realizar correctamente las técnicas, puesto que la gran mayoría de gestos técnicos son multiarticulares e intervienen ambos lados corporales, tanto de piernas como de brazos de 
manera simultánea, combinada y coordinada. En un estudio desarrollado por Emre, Gursoy, Ozan, Hazar y Dalli (2018) se observa un mayor éxito deportivo en practicantes de Karate zurdos que en diestros, y una revisión de la literatura parece sugerir que, en comparación con la incidencia en la población normal, los atletas zurdos están considerablemente sobrerrepresentados en el deporte, y de forma concreta en deportes interactivos uno contra uno (Loffing y Hagemann, 2016); esto podría indicar que los atletas zurdos disfrutan de una ventaja de rendimiento en estos deportes. En dicha revisión, no se considera la hipótesis de superioridad innata, sino que se considera que la ventaja de zurdos ante diestros en este tipo de disciplinas puede deberse principalmente, pero no exclusivamente, a la combinación de las demandas de los deportes interactivos uno a uno con la dificultad de encontrar soluciones óptimas contra oponentes del lado izquierdo que rara vez se encuentran (dependencia de frecuencia negativa). En el kumite, donde prima mucho la capacidad de sorprender al rival para obtener puntos, cabe pensar que el empleo equitativo de ambos lados corporales puede suponer una ventaja al dotar de mayor incertidumbre las intervenciones del Karateka, logrando que el rival no pueda anticipar con tanta facilidad los gestos empleados. Por otro lado, si un karateka posee un lado dominante extraordinariamente efectivo, sería lógico encontrar un uso más frecuente del mismo. En todo caso, parece que es claramente necesario en esta disciplina potenciar el trabajo del lado dominante y del no dominante, puesto que el deportista obtiene más cantidad de recursos técnicos y una mayor capacidad para resolver situaciones adversas de manera fluida y acertada durante los combates. Pese a ello, hay tendencia al empleo de un lado sobre otro en acciones de combate. Por ejemplo, según Lapresa, Ibáñez, Arana y Garzón (2011), los competidores de la categoría juvenil muestran un claro predominio en la utilización de guardia de izquierda, para atacar con técnica directa a la zona media, con puño dominante, mientras en categorías superiores se comprueba un empleo más equilibrado de las guardias. Esa tendencia a usar mayoritariamente el lado dominante, podría limitar la capacidad sorpresiva, por lo que podría determinar los resultados obtenidos en competiciones. En el caso de la modalidad kata, al tratarse de una serie de técnicas predeterminadas que no buscan un impacto en un objetivo real ni un efecto sorpresivo, no existen evidencias claras de un beneficio de un trabajo bilateral

Después de todo lo mencionado, queda evidenciada la importancia de la dominancia lateral en el rendimien- to en determinados deportes, que sin duda afecta a la forma de plantear sus entrenamientos; sin embargo, no hay apenas trabajos centrados en cómo enfocan los entrenadores/as sus intervenciones. En nuestro caso concreto, centramos nuestro trabajo en el Kárate, y pretendemos examinar la percepción de los entrenadores y entrenadoras del tratamiento de la lateralidad en el entrenamiento del karate.

\section{Metodología}

\section{Diseño del estudio}

Para la realización de este trabajo se ha empleado una metodología cuantitativa de corte descriptivo, llevándose a cabo un estudio transversal basado en la muestra. El estudio sigue los principios éticos de la «Declaración de Helsinki».

\section{Instrumento}

Establecidos los objetivos de trabajo, se creó un cuestionario ad hoc para proceder a la recogida de información, realizando posteriormente el procesamiento de datos, y el análisis los mismos, tal como indica el procedimiento de Campos Izquierdo, Martínez del Castillo, Mestre Sancho \& Pablos Abella (2007). El cuestionario se aplicaba de forma auto-administrada, en versión online. No se halló en la bibliografía ninguna herramienta validada que permitiese dar respuesta a nuestro objeto de estudio, por lo que se partió de cuestionarios relacionados con el tratamiento de la lateralidad otras disciplinas deportivas (Estrada-Marcén, Redondo-Laencina, Casterad-Seral \& Cid-Yagüe, 2021), y de estudios sobre la temática (Bilbao \& Oña, 2006; Mayolas Pi, 2011; Squadrone, Gallozzi \& Pasquini, 1995).

El cuestionario, diseñado al efecto en base a la temática de estudio y los objetivos planteados, pretendía recabar información sobre una muestra de la población estudiada con la finalidad de producir descripciones cuantitativas de diversos aspectos. Se contemplaban en el cuestionario preguntas tanto de tipo abierto (respuesta corta y párrafo) como cerrado (desplegables, selección múltiple y casillas de verificación), y también así cuestiones que pretendían contrastar el cumplimiento de los criterios de inclusión establecidos. Fue sometido a juicio de 3 expertos en Karate antes de aplicarlo para garantizar la comprensión y coherencia.

\section{Procedimiento}

Se contactó con los/as participantes a través de distintas redes sociales, informando sobre la finalidad del 
estudio, sobre los criterios de inclusión, y difundiendo el link a la encuesta. Se empleó la aplicación online Google Forms, incluida en la suite de oficina de Google Drive para aplicar el cuestionario. Esto permitió que se pudiera distribuir de forma rápida y sencilla, con un sistema fácil de acceso que favoreciera la participación. El link se enviaba junto con un breve mensaje explicando la finalidad del estudio, las características del cuestionario, informando sobre los criterios de inclusión, sobre el tiempo aproximado de cumplimentación, y sobre el tratamiento de los datos recabados con fines de investigación. Se incluyó una casilla de verificación previa al cuestionario para que el participante diera su consentimiento informado para el tratamiento de los datos para la finalidad descrita. Las vías empleadas para el envío del enlace al cuestionario fueron el correo electrónico para los contactos conocidos, y las aplicaciones Whatsapp y Facebook para la difusión a grupos de entrenadores/as.

\section{Participantes}

La población objeto de estudio eran entrenadoras y entrenadores en activo que estuviesen trabajando como técnicos de Kárate en España, y que desarrollasen su labor con cualquier nivel de competición federada. No se acotó edad, experiencia, ni formación

Se obtuvieron un total 72 respuestas de entrenadores/as de karate, de las cuales 50 corresponden a hombres y 22 a mujeres. En cuanto al promedio de edad, se registra un valor de 26,74 $\pm 8,17$ años. Aunque no estaba establecido como criterio de inclusión, todos poseen experiencia deportiva competitiva en el karate en distintos niveles.

\section{Análisis estadístico}

Las respuestas obtenidas en los cuestionarios de Formularios Google, se recogieron y organizaron automáticamente mediante el programa ofimático de Microsoft Excel en forma de tablas de datos. Posteriormente, dentro del mismo programa estadístico se analizó la información recolectada por medio de dos tipos de análisis. Por una parte, el análisis descriptivo, con el cual se obtienen frecuencias, porcentajes, medias y desviaciones típicas, en función de la naturaleza de los datos. Por otra parte, el análisis inferencial para correlacionar variables mediante la realización la prueba de Spearman, empleando un intervalo de confianza del 95\%, y estableciéndose como nivel de significación una $p<0.05$, empleando para ello el complemento de Excel XLSTATS.

\section{Resultados}

Al plantear a los entrenadores/as si, en su experiencia como deportistas en el Karate han experimentado en algún momento de su vida un intento por parte de algún entrenador por cambiar su lateralidad, la mayoría de encuestados (69\%), niega haber vivido esta situación, frente a un $28 \%$ que la han vivenciado. A aquellos que afirmaban haber sido forzados a un cambio de lateralidad, se les solicita que respondan si éste logró ser permanente en el tiempo y si se consiguió afianzar el cambio en su práctica deportiva cotidiana. En el caso de los que experimentaron un cambio de lateralidad permanente provocado por el entrenamiento, se les pide que seleccionen cuál fue el segmento corporal en el que se produjo la modificación. Hay en sus respuestas una evidente preponderancia del segmento podal (70\%) frente al manual (20\%), mientras sólo un 10\% mantiene que el cambio se produjo tanto en pie como en mano.

En relación a la frecuencia con la que los entrenadores/as trabajan de forma equilibrada ambos lados corporales en la modalidad de kata y en la de kumite, tanto en una como en otra la mayoría de entrenadores/as realiza un trabajo compensado de ambos lados corporales «casi siempre» o «siempre», tal como se aprecia en la figura 1. Al consultarles sobre la modalidad en la que consideran más importante trabajar de manera equilibrada lado derecho e izquierdo, los entrenadores/as se decantan mayoritariamente por la opción de que en ambas modalidades es igual de importante el trabajo bilateral equilibrado (50\%). Un 29\% se inclina por opinar que es más importante en Kumite, mientras un 21\% considera que en Kata-Kihon.

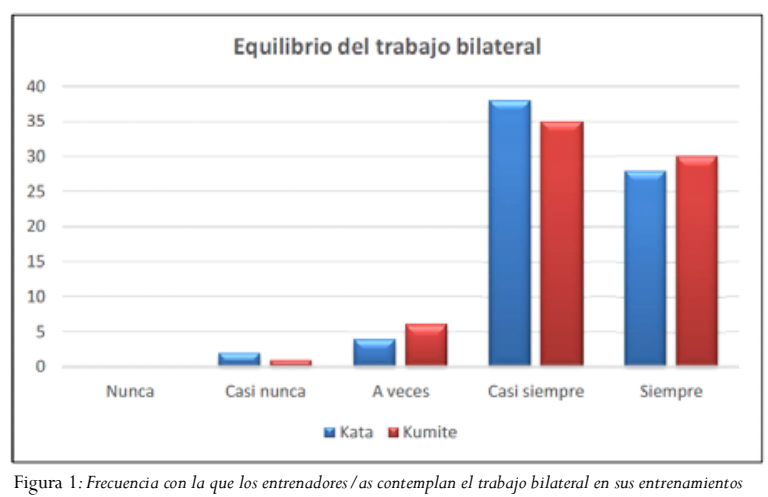

tanto en la modalidad de Kata como en la de Kumite.

También se les preguntó si habían intentado en alguna ocasión modificar la lateralidad de sus deportistas a través del entrenamiento; un 51\% de los entrenadores/as niega haber tratado de modificar la lateralidad de su alumnado, frente a un 49\% que sí lo ha intentado con alguno de ellos. Dentro de aquellos que sí han bus- 
cado el cambio, la mayoría manifiesta que éste no ha sido permanente (68\%). En relación al segmento corporal cuya lateralidad se pretendía modificar, la mayoría optó por seleccionar mayoritariamente la opción del pie. La modalidad en la que predomina el intento de modificación es el kumite con un 77\%, frente al 13\% de kata y al 10\% de ambas. Pese a que los entrenadores/as opinan que en ambas modalidades es igual de importante trabajar la bilateralidad, donde más intentos de cambio de lateralidad han llevado a cabo es en el kumite.

Además de solicitar información sobre el segmento y la modalidad para la que se pretendió cambiar la dominancia lateral de los karatekas, también se pregunta sobre cuál fue la percepción de dificultad subjetiva que experimentaron los entrenadores/as a la hora de planificar, diseñar y dirigir el entrenamiento específico. La mayoría de entrenadores / as consideran que llevar a cabo la planificación fue «difícil» y ningún entrenador piensa que fue «muy poco difícil».

De los 31 entrenadores/as que trataron de cambiar la lateralidad de algún deportista, 11 explicaron qué tipo de ejercicios o estrategias utilizaban para trabajarla o modificarla. Redactamos a continuación alguna de ellas:

- «Realizar combates sin poder utilizar los lados dominantes durante el mismo.»

- «Ejercicios de disociación y ejercicios sin utilizar el lado dominante».

- «Suprimir el lado dominante en Jiyu kumite».

- «Uso entrenamientos que supriman el uso de las técnicas con el lado dominante y que potencien el lado menos utilizado durante los combates o el menos hábil».

- «Tanto en kata como en kumite insisto en que se hagan la misma cantidad de repeticiones con ambos lados, aunque hay veces que según el tiempo de clase tengo que optar por trabajar solo un lado. En ese caso intento que trabajen su lado no dominante».

- «Para combate suelo hacer que no utilicen su lado dominante en Jiyu kumite, o que vayan cambiando cada cierto tiempo el lado que pueden utilizar. Para kata Hago kihon se trabaja en la misma proporción los dos lados».

- «Ejercicios disociativos así como ejercicios exclusivamente con el miembro nodominante».

- «En kumite trabajo más con compañeros y con trabajo de reflejos en los que usen sólo uno de los lados del cuerpo que yo especifique, y en kata trabajo alternativamente los dos lados».

- «Trabajo prohibiendo el uso de un lado u otro de manera alternada».

Al solicitar a los participantes que manifiesten su opinión en relación a si consideran adecuado cambiar la lateralidad de un karateka con fines competitivos, la mayoría considera correcto que la lateralidad se modifique por este motivo (69\%). Los entrenadores/as también ofrecen una respuesta frente a la cuestión de cuál es el nivel competitivo que ellos consideran más adecuado en el caso de que tuvieran que realizar una alteración de la lateralidad de algún karateka. La mayoría de ellos creen que el nivel más apropiado es el Nacional (57\%), un 24\% opina que en todos los niveles podría ser adecuado, un 11\% en Autonómico, y un 8\% en Escolar.

Ante la cuestión sobre si ven adecuado tratar de modificar la lateralidad de sus deportistas a una edad determinada, un $21 \%$ provocarían el cambio a cualquier edad, frente a un 79\% que sólo lo haría a determinadas edades. Y en relación a ello, se presenta la figura 2, donde manifestaban cuál era la categoría que estimaban más adecuada para realizar cambios en la lateralidad de sus karatekas. Los entrenadores/as consideran más adecuado trabajar los cambios en un rango de edad que abarca desde los 8 a los 13 años.

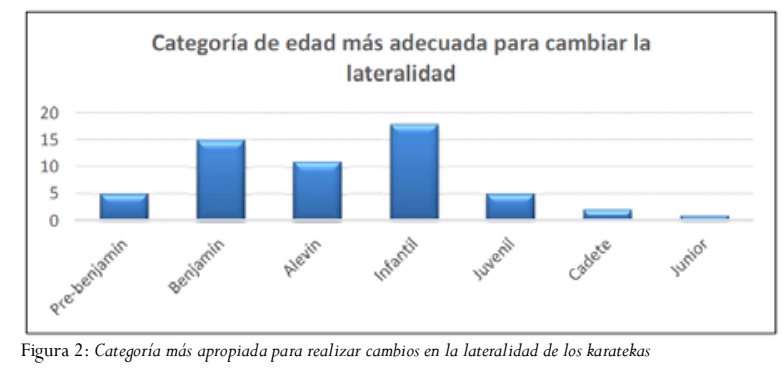

\section{Discusión}

Existe un predominio de los entrenadores/as que niegan que los cambios producidos en su lateralidad en su época como deportistas se mantuvieran en el tiempo $(60 \%)$. Y en relación a la durabilidad de los cambios de lateralidad forzados como entrenadores/as en sus deportistas, la mayoría afirma que los cambios logrados tampoco han sido permanentes. Es necesario tener en cuenta que, aunque aún no existe absoluta certeza sobre el factor o factores que determinan nuestro tipo de lateralidad (Vallortigara y Rogers, 2005), alrededor de este asunto surgen dos teorías que, aunque parecen irreconciliables, resultan ser complementarias. Aunque hay autores que apuestan por un origen ambiental de la lateralidad (Kumar y Mandal, 2005, Teixeira, Silva y Carvalho, 2003, Van Mier, Perlmutter y Petersen, 2004, Judge y Stirling, 2003), hay otras teorías que abogan por un origen genético de la misma (Laland, Kumm, Van Horn y Feldman, 1995, Yeo, Gangestad, Thoma, 
Shaw y Repa, 1997, McManus, 1985, Annet, 1998, Gunhaga, Jessell y Edlund, 2000, Francks, Maegawa, Laurén, 2007), y el gran peso de ese condicionante genético podría estar en la base de que los cambios producidos en la lateralidad de sus deportistas mediante el entrenamiento no se mantengan en el tiempo. Además, no siempre es posible modificar la dominancia lateral por motivos orgánicos o funcionales, como podría ser un patrón de organización lateral muy estructurado (Bernabeu, 2016), lo que podría también justificar estos cambios temporales y no permanentes. También en relación a esto, hay estudios que afirman que la dominancia podría entenderse como una particularidad muy asociada a una determinada tarea en vez de tratarse de un fenómeno general a nivel de una extremidad completa (Vagenas y Hoshizaki, 1991), por lo que quizá sería interesante diferenciar en futuros trabajos si los cambios buscados y/o logrados se centran en la dominancia a nivel global de la extremidad o a nivel de tarea.

La gran mayoría de los entrenadores que en su época como deportistas modificaron de forma permanente la lateralidad de un segmento corporal, experimentaron dicho cambio a nivel podal (70\%). Este dato podría ser debido a que el Kárate es una disciplina en la que no se realiza manipulación de objetos. En otros deportes como la gimnasia rítmica, donde existe manipulación, las entrenadoras manifiestan mayor equilibrio entre cambios de lateralidad provocados en pies y en manos (Estrada-Marcén et al., 2021).

La gran mayoría de participantes realiza un trabajo compensado de ambos lados corporales en Kata y en Kumite. Ya a finales de la década de los 80, Starosta (1988) evidenció la relevancia del trabajo simétrico entre lado izquierdo y derecho en los jugadores de fútbol, avalando en otros deportes la importancia que también los participantes en nuestro estudio otorgan al entrenamiento compensado. En un estudio reciente realizado en Karatekas (Honorato, Mattos, Torres, Oliveira y Banja, 2021) se evaluaron el tiempo de reacción, el tiempo de respuesta, la velocidad del golpe y la patada, además de la fuerza máxima del golpe, tanto en brazo como en pierna, y fueron halladas diferencias significativas entre miembro dominante y no dominante en todas las medidas de resultado, lo que evidencia una asimetría funcional que puede producir una desventaja competitiva, con la necesidad de atención en el entrenamiento que eso conlleva. No obstante, Oliveira, Barbieri, Gobbi y Drigo (2008), mantienen que la práctica regular con ambos lados desde las primeras etapas del aprendizaje promueve la simetría entre las extremidades en la patada Mae-
Geri en Karatekas, eliminando la especificidad de cada extremidad, por lo que un entrenamiento bien planificado puede evitar asimetrías funcionales.

Gran parte de los participantes ven el trabajo bilateral equilibrado igual de importante en ambas modalidades. Aunque en Kumite se puede ver claramente la ventaja del trabajo bilateral de cara a ganar ventaja sorpresiva frente al contrincante y capacidad de adaptación a las acciones del rival, en el caso de la modalidad kata, posibles desequilibrios de técnica, fuerza o coordinación que sean muy llamativos entre ambos lados corporales podrían deteriorar la calificación global final de la ejecución.

Prácticamente la mitad de la muestra manifiesta haber tratado de modificar la lateralidad de sus deportistas a través del entrenamiento. Los deportistas zurdos parecen estar en situación de ventaja en deportes individuales de oposición uno contra uno (Bejarano y Naranjo, 2014, Gutwinski, Löscher, Mahler, Kalbitzer, Heinz, y Bermpohl, 2011, Wood y Aggleton, 1989), como es el caso concreto del Karate, sin poderse concretar si esta superioridad se debe a la naturaleza del juego o a ventajas neurológicas.

Un gran porcentaje de los participantes manifiesta ser contrario a provocar cambios de dominancia lateral a determinadas edades, lo que podrían explicar que el porcentaje que pretende modificar la lateralidad de sus deportistas no sea más elevado de lo que arrojan los resultados. La modalidad en la que predomina el intento de modificación de lateralidad es el kumite (77\%). El kumite es una modalidad interindividual, y una escasez de habilidad en un lado puede limitar en gran medida los resultados competitivos, por lo que puede ser determinante poseer una capacidad técnica muy trabajada en ambos lados. Ya hemos visto que se observa un mayor éxito deportivo en practicantes de Karate zurdos (Emre et al., 2018), aunque cuando se habla de esta ventaja, no se especifica la modalidad en la que tiene lugar. Pero hay estudios que hablan de una mayor probabilidad de éxito de los zurdos en deportes de uno contra uno (Dopico-Calvo, Iglesias-Soler, Morenilla, Giráldez, Santos y Ardá, 2016), encontrándose la modalidad Kumite en esa clasificación. Quizá en la modalidad Kata, que es de participación individual, los entrenadores/as encuentran estrategias para solucionar la falta de equilibrio bilateral de sus deportistas, al igual que ocurría en el estudio de Estrada-Marcén et al. (2021).

En cuanto a la percepción de dificultad subjetiva que experimentaron para planificar, diseñar y dirigir el entrenamiento dirigido al cambio de lateralidad, a pesar 
de que cada vez está más claro que un cambio en la dominancia lateral de un deportista es más entrenable de lo que se había creído (Bilbao y Oña, 2000; Haaland y Hoff, 2003; Bell y Gabbard, 2000; Dörge, Bullandersen, Sorensen y Simonsen, 2002; Nicholls, Loetscher y Rademacher, 2010; Palau, López-Montero y López-Botella, 2010; Roberts yTurnbull, 2010; Dorochenko, 2013; Koch y Bozzali, 2011), no hay evidencias científicas que se centren en cómo deben ser los entrenamientos en las distintas disciplinas para lograr cambios en esa dominancia lateral, por lo que muchos entrenadores/as no encuentran directrices a seguir al planificar su entrenamiento. Esto podría dificultar su labor e incluso ser una de las causas de que descartasen tratar de modificar la dominancia lateral de sus deportistas.

Los participantes han referido numerosas estrategias para tratar de modificar la lateralidad de sus deportistas. En estudios como el de Del Valle y De la Vega (2007), basados en otro deporte de combate como es el Full Contact, ya se resaltaba la importancia que adquiere el protocolo de actuación seguido por el entrenador con vistas al uso de una «bilateralidad». Se puede apreciar a partir de los testimonios de los entrenadores/as que las estrategias empleadas son similares en la mayor parte de los casos, realizando supresión en las técnicas hacia un lado o hacia otro, con el fin de que se practiquen ambos lados por igual y equilibrando la destreza bilateral. Se implementan ejercicios de carácter disociativo para adquirir maestría en los gestos técnicos independientemente del lado con el que se realicen. Tanto en kata como en kumite se busca trabajar con un número parecido de repeticiones con ambos lados corporales. Cabe destacar que ningún entrenador/a menciona el hecho de proporcionar feedback positivo o algún tipo de refuerzo cuando los deportistas realizan correctamente un gesto con su lado no dominante, siendo ésta una técnica de modificación de conductas motrices que, según Bilbao y Oña (2000), resulta muy efectiva para desarrollar el uso, el conocimiento y la elección correcta de los lados corporales en los trabajos de aprendizaje motor. En todo caso, según Vidranski, Ma skarin y Juki (2015), las peleas de kárate se ganan utilizando principalmente técnicas con bajos requisitos biomecánicos, por lo que podría ser una estrategia para los entrenadores, sobre todo en edades tempranas (aunque una vez con consolidada la lateralidad), comenzar el trabajo para lograr el equilibrio de la destreza bilateral sólo en técnicas sencillas. En relación al desempeño lateral en kárate formativo en la modalidad kumite, un reciente estudio traza una hoja de ruta para el entrena- miento hacia la equilateralidad enfocada hacia la competición de élite (Sastre-Fácila, Lapresa-Ajamil AranaIdiakez, Ibáñez y Anguera, 2021), indicando que el karateka de 8-9 años debe superar una prevalencia lateral condicionada, adoptando una pierna delantera no dominante para luego atacar con el segmento corporal dominante, y que el uso equilibrado del puño derecho o izquierdo debe ocurrir más tarde, entre el grupo de edad de 12-13 años, habiendo entonces menos condicionamiento lateral decisivo en la ejecución de técnicas ofensivas de piernas.

La gran mayoría justifica que se cambie la lateralidad de los deportistas con fines competitivos, sobre todo a partir del nivel de competición nacional. En el estudio de Estrada et al. (2021) centrado en la Gimnasia Rítmica, disciplina en la que una determinada dominancia lateral no parece condicionar en tanta medida como en deportes de adversario el rendimiento final, un $52 \%$ de las entrenadoras modificarían la dominancia de las gimnastas con fines competitivos. Los resultados en el mencionado estudio son similares a los nuestros también en cuanto a los niveles en los que forzarían dicho cambio, encontrando que un $44 \%$ sólo lo harían a partir de nivel Nacional, es decir, a un nivel competitivo bastante alto. Esto podría ocurrir porque, llegados a este nivel, cobran más relevancia los resultados que en niveles inferiores (Estrada et al., 2021).

Un 79\% de participantes sólo provocaría el cambio de la lateralidad de sus deportistas a determinadas edades, y no en cualquier momento, y encuentran como el momento más adecuado para hacerlo el rango de edad entre los 8 y los 13 años. Hay que resaltar que a partir de los 8 años se considera consolidada la lateralidad ocular, auditiva, podal y manual (Castañer y Camerino, 1993: López-Sánchez, 2004) mostrando a estos niveles un predominio tanto cuantitativo como cualitativo. $\mathrm{Si}$ bien es verdad que se relacionan las etapas de desarrollo infantil con una mayor capacidad de las personas para recibir aprendizajes de tipo motriz (Gesell, 1958), y que se podría pensar que cuanto más temprano se provoquen los cambios, más fácil será que éstos los adquieran y consoliden, se debería tender en el entrenamiento deportivo a enseñar potenciando la bilateralidad o los posibles cambios de ésta, siempre y cuando la lateralidad del niño esté consolidada (Castañer y Camerino, 2006), es decir, a partir de los 7-8 años. Las preferencias laterales se modifican, probablemente debido a la neuroplasticidad, durante la adquisición de habilidades motoras y posturales en el entrenamiento deportivo a largo plazo (Mikheev et al., 2002), y a esas 
edades en torno a los 8 años, la plasticidad cerebral sigue siendo alta, puesto que el rango de edad en el que la plasticidad aparece es amplio, aunque pobremente delimitado (Lenn, 1992).

\section{Conclusiones}

El ambidextrismo es, tanto en la modalidad de kumite como en la de kata, el tipo de lateralidad que más ventajas aporta a nivel competitivo según los entrenadores/as. El segmento en el que es más común que los deportistas sufran un cambio de lateralidad forzada por el entrenamiento es el pie. Dicha modificación suele producirse mayoritariamente en aquellas personas que son diestras, con el objetivo de que cambien a una dominancia lateral zurda.

Los cambios de lateralidad permanentes en los practicantes de Karate llevados a cabo por la intervención de sus entrenadores/as son «poco frecuentes». La mayoría de los entrenadores/as que intentar cambiar la lateralidad de sus deportistas, lo hace cuando tienen en torno a 10-11 años, aunque también hay un importante número de casos que lo hace entre los 7 y 9 años, y por norma general cuando los alumnos se encuentran en nivel competitivo nacional. La mayoría de entrenadores/as considera inadecuado realizar el cambio a cualquier edad y que cree que se debe procurar realizar el cambio en torno a las edades mencionadas. La modalidad en la que se busca en mayor medida el cambio de lateralidad es en kumite.

La mayoría de los entrenadores/as de karate se muestran a favor de cambiar la lateralidad de sus alumnos alegando fines competitivos, aunque consideran que realizar la planificación del entrenamiento con el fin der lograr un cambio en la lateralidad es «bastante difícil». Los entrenadores/as de karate «casi siempre» realizan un trabajo bilateral equilibrado tanto en kumite como en kata y consideran importante que se mantenga este balance.

\section{Declaración de disponibilidad de datos}

Datos disponibles a petición de los autores.

\section{Referencias}

Annet, M. (1998). Handedness and cerebral dominance: The right shift theory. The Journal of Neuropsychiatry and Clinical Neurosciences, 10(4), 459-69. DOI: 10.1176/jnp.10.4.459
Bejarano, M. \& Naranjo, J. (2014). Lateralidad y rendimiento deportivo. Archivos de Medicina del Deporte, 31(3), 200-204.

Bell, J. \& Gabbard, C. (2000). Foot preference changes through adulthood. Laterality, 5, 63-8. DOI: 10.1080/ 713754351

Bernabeu, E. (2016). Programas de desarrollo de la lateralidad, mejora del esquema corporal y organizacion espaciotemporal. Intervencion en dificultades de aprendizaje, en Procesos y programas de Neuropsicología Educativa (Ministerio Educación Cultura y Deporte).

Bilbao, A. \& Oña, A. (2000). La lateralidad motora como habilidad entrenable. Efectos del aprendizaje sobre el cambio de tendencia lateral. Motricidad, 6, 7-27. DOI: 10.21134/eurjhm.2000.6.52

Bolte, J.T. (2009). My Stroke of Insight: A Brain Scientist's Personal Journey. Londres: Plume Editions.

Castañer, M. \& Camerino, O. (1993). La Educación Física en la Enseñanza Primaria. Barcelona: Ed. Inde.

Castañer, M. \& Camerino, O. (2006). Manifestaciones básicas de la motricidad. Lleida: Edicions de la Universitat de Lleida.

Del Valle Díaz, S. \& De la Vega Marcos, R. (2007). Lateralidad en el deporte de Full Contact. Cambios en diferentes condiciones. Revista Internacional de Medicina y Ciencias de la Actividad Física y del Deporte, $7(25), 32-51$

Dopico-Calvo, X, Iglesias-Soler, E., Morenilla, L, Giráldez, M.A, Santos, L. \& Ardá, A. (2016). Laterality and performance in combat sports. Archives of Budo: Science of Martial Arts, 12, 167-177.

Dörge, H.C, Bullandersen, T, Sorensen, H. \& Simonsen, E.B. (2002). Biomechanical differences in soccer kicking with the preferred and the non-preferred leg. Journal of Sports Sciences, 20, 293-9. DOI: 10.1080/026404102753576062

Dorochenko, P. (2013). El ojo director. Paul Dorochenko Ed. (edición kindle, Amazon.es).

Emre, Y, Gursoy, R, Ozan, M, Hazar, K. \& Dalli, M. (2018). Research on the Relation between Hand Preference and Success in Karate and Taekwondo Sports with Regards to Gender. Advances in Physical Education, 8(3), 308-320. DOI: 10.4236/ ape. 2018.83027

Estrada-Marcén, N.; Redondo-Laencina, P.; CasteradSeral, J. \& Cid-Yagüe, L. (2021) Laterality Treatment in Rhythmic Gymnastics: Research Based in Coaches. Revista Internacional de Medicina y Ciencias de la Actividad Física y el Deporte, 21(84), 479-500. DOI: https: / 
/doi.org/10.15366/rimcafd2021.83.005

Ferré, J. \& Irabau, E. (2002). El desarrollo neurofuncional del niño y sus trastornos. Madrid: Lebón.

Franchini, E, Sterkowicz, S, Meira, C.M Jr, FerreiraGomes, F.R. \& Tani, G. (2008). Technical variation in a sample of high level judo players. Perceptual and Motor Skill, 106(3), 859-869. DOI: 10.2466/ pms.106.3.859-869

Francks, C, Maegawa, S. \& Laurén, J. (2007). LRRTM1 on chromosome $2 \mathrm{p} 12$ is a maternally suppressed gene that is associated paternally with handedness and schizophrenia. Molecular Psychiatry, 12, 1129-39. doi:10.1038/sj.mp.4002053

Gunhaga, L, Jessell,T. \& Edlund,T. (2000). Sonic hedgehog signaling at gastrula stages specifi es ventral telencephalic cells in the chick embryo. Development. 127, 3283-93.

Gutwinski, S, Löscher, A, Mahler, L, Kalbitzer, J, Heinz, A. \& Bermpohl, F. (2011). Understanding lefthandedness. Deutsches Ärzteblatt International, 108(50), 849-53. DOI: 10.3238/arztebl.2011.0849

Haaland, E. \& Hoff, J. (2003). Non dominant leg training improves the bilateral motor performance of soccer players. Scandinavian Journal of Medicine \& Science in Sports, 13, 179-84. DOI: 10.1034/j.16000838.2003.00296.x

Hildreth, G. (1949) The developement and training of hand dominance. (En Lerbet, G., 1977). La lateralidad en el niño y en el adolescente: niños derechos y niños. España: Marfil.

Honorato, M.X., Mattos Frota de Souza, T, Torres Cabido, C.E., Oliveira Assumpcao, C. \& Banja,T. (2021). Assessment of strike time and laterality in karate athletes. Coleção Pesquisa em Educação Física, 20(3), 37 45.

Judge, J. \& Stirling, J. (2003). Fine motor skill performance in left and right handers. Evidence of an advantage for left handers. Laterality, 8(4), 297-306. DOI: $10.1080 / 13576500342000022$

Koch, G. \& Bozzali, M. (2011). Asymmetry of parietal interhemispheric connections in humans. Journal of Neurosciences, 31(24):8967-75. DOI: 10.1523/ JNEUROSCI.6567-10.2011

Kumar, S. \& Mandal, M.K. (2005). Bilateral transfer of skill in left and right handers. Laterality, 10(4), 33744. DOI: $10.1080 / 13576500442000120$

Laland, K.N, Kumm, J, Van Horn, J.D. \& Feldman, M.W. (1995). A gene-culture model of human handedness. Behavior Genetics, 25, 433-45. DOI: 10.1007/ BF02253372
Lapresa, D, Ibáñez, R, Arana, X. \& Garzón, B. (2011). Estudio comparativo de las acciones de combate en el karate de categoría juvenil (12-13 años) y sénior. Apunts. Educacion Fisica y Deportes, 2(104): 66-79. DOI: 10.5672/apunts.2014-0983.es.(2011/2).104.07

Lenn, N.J. (1992). Brain plasticity and regeneration. American Journal of Neuroradiology, 13: 505-5

Lerbet, G. (1977). La lateralidad en el niño y en el adolescente: niños derechos y niños zurdos. Alcoy, España: Marfil.

Loffing, F. \& Hagemann, N. (2016). Performance differences between left- and right-sided athletes in one-on-one interactive sports. In F. Loffing, N. Hagemann, B. Strauss, \& C. MacMahon (Eds.), Laterality in sports: Theories and applications (249-277). Elsevier Academic Press. https: / / doi.org/10.1016/ B978-0-12-801426-4.00012-2

López Sánchez, J.M. (2004). Los contenidos de la Educación Física en la Educación Primaria. Granada: Grupo Editorial Universitario.

Magill, R. A. (1993). Motor Learning: concepts and applications. Iowa. Wm C Brown, $4^{\text {a }}$ edición. (En Bejarano, M. \& Naranjo, J. (2014). Lateralidad y rendimiento deportivo. Archivos de Medicina del Deporte, 31(3), 200-204.

McManus, I.C. (1985). Handedness, language dominance and aphasia: a genetic model. Psychological Medicine, $8,1-40$.

Mikheev, M, Mohr, C, Afanasiev, S, Landis, T. \& Thut, G. (2002). Motor control and cerebral hemispheric specialization in highly qualified judo wrestlers. Neuropsychologia, 40, 1209-19. DOI: 10.1016/S00283932(01)00227-5

Nicholls, M.E.R, Loetscher,T. \&Rademacher, M. (2010). Miss to the Right: The Eff ect of Attentional Asymmetries on Goal-Kicking. PLoS ONE, 5(8), e12363. DOI: 10.1371/journal.pone.0012363

Oliveira, L.; Barbieri, F.; Gobbi, L. \& Drigo, A. (2008). Simetria intermembros no desempenho do chute maegeri do karatê. Rev. Bras. Ciênc. Mov, 16(4), 1-18, https: / / doi.org/10.18511/rbcm.v16i4.901.

Palau, J.M, López-Montero M. \& López-Botella M. (2010). Relación entre eficacia, lateralidad, y zona de lanzamiento del penalti en función del nivel de competición en el fútbol. International Journal of Sport Sciences, 19(6):154-65. DOI: 10.5232/ ricyde2010.01905

Pieron, H. (1968) Vocabulaire de la Psychologie. Paris: PUF. (En Lerbet, G., 1977). La lateralidad en el niño y en el adolescente: niños derechos y niños zurdos. 
Alcoy,

Roberts, R. \& Turnbull, OH. (2010). Putts that get missed on the right. Investigating lateralized attentional biases and the nature of putting errors in golf. Journal of Sports Sciences, 28(4), 369-74. DOI: 10.1080/02640410903536467

Sainburg, R. (2016). Laterality of Basic Motor Control Mechanisms: Different Roles of the Right and Left Brain Hemispheres. Laterality in Sports: Theories and Applications, Chapter 8.

Sastre-Fácila, V, Lapresa-Ajamil, D, Arana-Idiakez, X, Ibáñez, R. \& Anguera, MT (2021). Observational Analysis of Lateral Preference in Kumite Initiation: A Starting Point in the Longitudinal Programming of Formative Karate. Perceptual and Motor Skills, 128(6), 1-14. DOI: 10.1177/00315125211039198

Sogabe, A., Sterkowicz-Przybycieñ, K., Maehara, K., Sasaki, T. \& Sterkowicz, S. (2015). Effect of preferred body stance side on the performance of Special Judo Fitness Test in Japanese judo athletes. Archives of Budo: Science of Martial Art, 11, 1-6.

Starosta, W. Symmetry and asymmetry in shooting demonstrated by elite soccer players.In: Science and Football. London. E \& FN Spon; 1988.

Sterkowicz, S., Lech, G. \& Blecharz, J. (2010). Effects of laterality on the technical/tactical behavior in view of the results of judo fights. Archives of Budo: Science of Martial Art, 6(4), 173-177.

Teixeira, L.A, Silva, M.V.M. \& Carvalho, M. (2003).
Reduction of lateral asymmetries in dribbling. The Role of Bilateral Practice.Laterality, 8(1), 53-65. DOI: 10.1080/713754469

Vagenas, G. \& Hoshizaki, B. (1991). Functional Asymmetries and Lateral Dominance in the Lower Limbs of Distance Runners. International Journal of Sport Biomechanics, 7, 311-29. DOI: https://doi.org/ 10.1123/ijsb.7.4.311

Vallortigara, G. \& Rogers, L.J. (2005). Survival with an asymmetrical brain: advantages and disadvantages of cerebral lateralization. Behavioral and Brain Sciences, 28, 575-633. DOI: 10.1017/S0140525X05000105.

Van Mier, H.I, Perlmutter, J.S. \& Petersen, S.E. (2004). Functional Changes in Brain Activity During Acquisition and Practice of Movement Sequences. Motor Control, 8, 500-20. DOI: 10.1123/mcj.8.4.500.

Vidranski,T., Maskarin, F. \& Jukic, J. (2015). Differences in technical and tactical indicators of attacks and counterattacks in elite male karate fighters. Acta Kinesiologica, 9(1), 19-24.

Wood, C.J. \& Aggleton, J.P. (1989). Handedness in fast ball sports. Do lefthanders have an innate advantage? British Journal of Psychology, 80, 227-4. DOI: 10.1111/ j.2044-8295.1989.tb02316.x

Yeo, R.A, Gangestad, S.W, Thoma, R.J, Shaw, P.K. \& Repa, K. (1997). Developmental instability and cerebral lateralization. Neuropsychology, 11, 552-61. zurdos. Alcoy, España: Marfil.

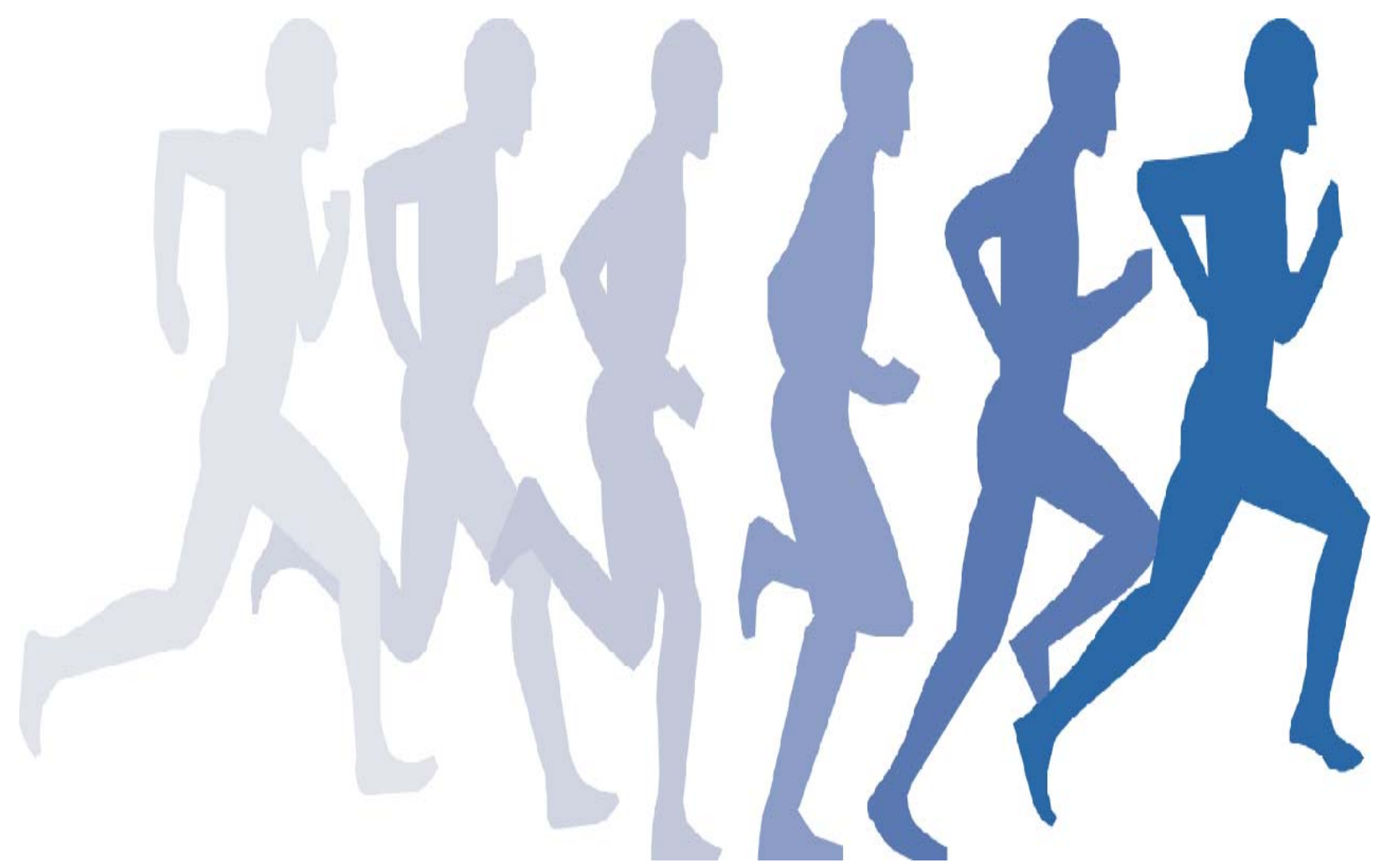

